\title{
Enfermedad de Grover tipo poroqueratósico: variante histopatológica a considerar
}

\section{Porokeratosis-like Grover's disease: Histopathological variant to consider}

\author{
Víctor M. Gutiérrez-San Lucas, Carmen López-Acosta y Isabel Talledo-Mera*,
} Servicio de Dermatología, Hospital de Especialidades “Teodoro Maldonado Carbo", Guayaquil, Ecuador

\begin{abstract}
Resumen
La enfermedad de Grover o dermatosis acantolitica transitoria es una entidad de causa desconocida y se caracteriza por pápulas o papulovesículas pruriginosas casi siempre localizadas en el tronco anterior y posterior. Su diagnóstico debe confirmarse mediante análisis histopatológico. Se presenta el caso de una mujer de 27 años de edad con lesiones papulovesiculares agrupadas en el tronco y la región proximal de extremidades, sometida a estudio histopatológico que confirmó el diagnóstico de enfermedad de Grover, en este caso de tipo poroqueratósico, una variante histológica atípica e infrecuente.
\end{abstract}

Palabras clave: Similar a poroqueratósico. Enfermedad de Grover. Acantólisis. Disqueratosis. Dermatosis acantolítica transitoria. Anatomopatología.

\begin{abstract}
Grover's disease or transient acantholytic dermatosis, is an entity of unknown etiology, characterized by the presence of pruritic papules or papulovesicles generally located in the anterior and posterior trunk. Its diagnosis must be confirmed by histopathological analysis. We present a 27-year-old, woman with papulovesicular lesions grouped in the trunk and proximal region of the extremities, who underwent a histopathological study that confirmed the diagnosis of Grover's disease, in this case a porokeratosis-like type, a non-classical histological type, and little frequent.
\end{abstract}

Key words: Porokeratosis-like. Grover disease. Acantholytic. Dyskeratosis. Transient acantholytic dermatosis. Anatomopathology.

\section{Introducción}

La enfermedad de Grover, descrita por primera vez en 1970, es una dermatosis acantolítica y transitoria caracterizada por pápulas o papulovesículas pruriginosas y discretas, distribuidas sobre todo en el tronco. La patogenia y la etiología de esta afección no son claras aún, pero muchos autores las han relacionado con el calor, sudoración, xerosis, psoriasis y queratosis solares. El diagnóstico de esta dermatosis se basa en la correlación clínico-patológica. El hallazgo histopatológico principal es la acantólisis y disqueratosis. Se han descrito cuatro patrones acantolíticos principales: Hailey-Hailey, similar a Darier (el más común), espongiótico y similar al pénfigo. En las publicaciones médicas se han notificado otros tipos histológicos adicionales infrecuentes, como la variante poroqueratósica, por lo que su diagnóstico patológico representa un desafío.
Correspondencia:

*Isabel Talledo-Mera

E-mail: talledoisabel89@gmail.com

0210-5187/@ 2020 Colegio Ibero Latinoamericano de Dermatología A.C. (CILAD). Publicado por Permanyer. Este es un artículo open access bajo la licencia CC BY-NC-ND (http://creativecommons.org/licenses/by-nc-nd/4.0/). 


\section{Caso clínico}

Paciente femenina de 27 años de edad, con fototipo de piel II, sin antecedentes patológicos personales y familiares de importancia; acude al servicio de dermatología por presentar lesiones cutáneas y prurito de 7 años de evolución. La exploración física mostró pápulas eritematosas y vesículas, algunas agrupadas y excoriadas, localizadas en tronco y parte proximal de las extremidades (Fig. 1A-C); la paciente refiere períodos de remisión y exacerbación espontáneos. La dermatoscopia reveló un fondo blanco rosado con microfisuras superficiales, descamación blanquecina y un tapón queratósico amarillo-marrón central (Fig. 1D). Ante sospecha clínica de enfermedad de Grover se realizó biopsia en sacabocados para estudio histopatológico que mostró una capa córnea compacta con focos de paraqueratosis, epitelio con acantosis discreta y espongiosis leve, además de acantólisis suprabasal focal y discreta cercana a las zonas de paraqueratosis (Fig. 2). La clínica y la histología establecieron el diagnóstico de enfermedad de Grover de tipo poroqueratósico.

\section{Discusión}

Ralph Wier Grover acuñó el término "disqueratosis acantolítica transitoria" en 1970 para describir un trastorno papulovesiculoso primario, que se manifiesta en la forma de pápulas pruriginosas en el tronco y las extremidades. En la actualidad se prefiere el término enfermedad de Grover para describir esta afección ${ }^{1,2}$. Scheinfeld, et al. describieron más adelante tres formas clínicas diferentes: transitoria, persistente y crónica asintomática ${ }^{3,4}$.

La enfermedad de Grover es una dermatosis papular pruriginosa, que en clínica se manifiesta con pápulas, pápulo-vesículas, algunas excoriadas ubicadas por lo general en tronco y parte proximal de las extremidades, aunque se han informado casos de afectación extensa 0 atípica ${ }^{5}$. El prurito puede ser leve, moderado 0 intenso y puede producir alteración del sueño y agravarse por el calor ${ }^{6}$. Se desconoce aún su etiología, pero muchos autores la han relacionado con la sudoración, el calor y el eccema. Se ha planteado que, en lugar de deberse al calor o la sudoración, esta dermatosis surge en el contexto de una epidermis intacta pero xerótica con disminución de la producción de sudor y es probable que se vincule con una integridad epidérmica deteriorada ${ }^{3}$. En una revisión sistemática en la que se incluyeron 69 casos se observó que las

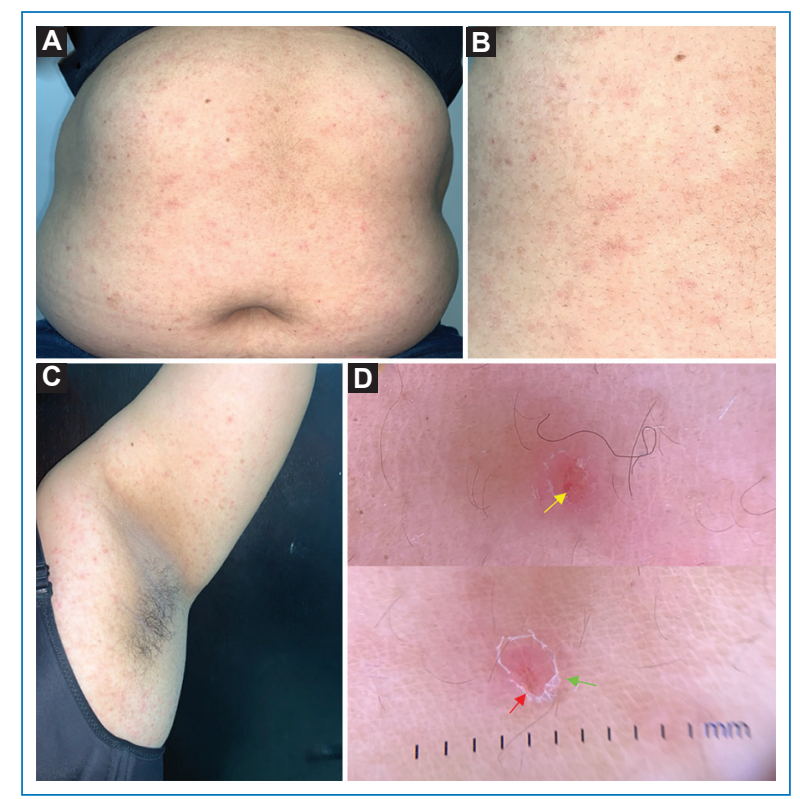

Figura 1. Fotografías clínicas del paciente.

A: papulovesículas eritematosas, algunas agrupadas y excoriadas localizadas en el abdomen. B: agrupación de pápulas a mayor aumento. C: algunas en la región proximal de las extremidades. D: dermatoscopia en la que se observan microfisuras superficiales (flecha amarilla), áreas escamosas (flecha verde) y un tapón queratósico marrón-amarillo central (flecha roja).

relaciones más comunes incluyeron antecedentes de proceso maligno $(61 \%)$, quimioterapia $(38 \%)$ y trasplante reciente $(20 \%)^{7}$.

Los hallazgos en la dermatoscopia orientan en esta dermatosis acantolítica, los mismos que dependen de la fase evolutiva de la lesión. En las primeras etapas, las erosiones superficiales y los vasos glomerulares centrales, lineales y en horquilla ubicados en la periferia corresponden a infiltrados inflamatorios perivasculares superficiales leves, mientras que en fases tardías estos vasos se muestran más prominentes, rodeados de un halo blanquecido que corresponde a las crestas alargadas, junto con un tapón queratósico amarillo-marrón central que se correlaciona con una acantólisis suprabasal, disqueratosis y acantosis ${ }^{8}$. También se han demostrado diferentes características según sea el subtipo histológico, pero muchas variantes poco frecuentes no se han comunicado. Un área central en forma de estrella, poligonal, ramificada o redondeada-ovalada, pardusca y rodeada por un halo blanquecino es característica del subtipo histológico similar a Darier, y la descamación blanquecina con un fondo rojizo-amarillento es típica del subtipo histológico 


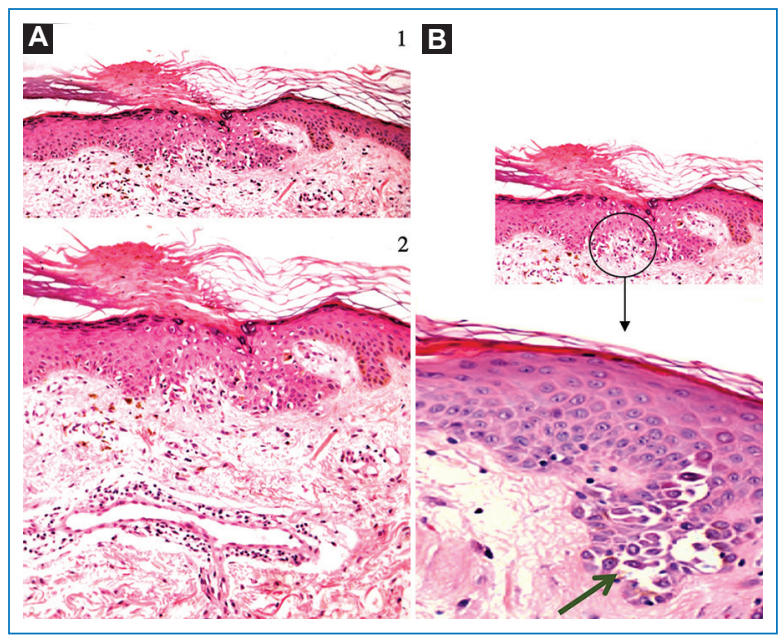

Figura 2. Estudio histopatológico. A: 1. Imagen panorámica de una lámina de paraqueratosis $(\mathrm{H}-\mathrm{E}$, 4x). 2. A mayor aumento: capa córnea compacta con focos de paraqueratosis que forman discretas lamelas y epitelio con acantosis discreta y espongiosis leve.

B: a mayor aumento (círculo) se observa epitelio con acantólisis suprabasal focalmente cercano a las zonas de paraqueratosis (flecha) (H-E, 10x).

espongiótico; en ambas formas se pueden encontrar vasos punteados o lineales irregulares ${ }^{9}$.

La correlación clínico-patológica de esta entidad es de suma importancia para un adecuado diagnóstico. En términos histopatológicos, el hallazgo más común es la acantólisis relacionada o no con disqueratosis. Grover al describir esta dermatosis mencionó dos patrones histopatológicos característicos: similar a Darier o Hailey-Hailey. En 1976, Simon, Bloom y Ackerman reconocieron dos variantes histopatológicas adicionales: el pénfigo vulgar (o foliáceo) y la forma espongiótica².

Fernández-Figueras, et al. ampliaron estos patrones, además de los hallazgos histológicos típicos ya descritos, y detectaron cinco patrones atípicos: poroqueratósico, vesicular, lentiginoso, liquenoide y dismaturativo con queratinocitos atípicos, lo que sugiere que los criterios del diagnóstico histopatológico de esta entidad deben ampliarse ${ }^{10}$.

El patrón poroqueratósico es una variante histopatológica infrecuente (menos del $5 \%$ de las series de casos), que se caracteriza por columnas oblicuas de paraqueratosis sobre áreas de epidermis que carecen de capa granular, con células disqueratósicas ocasionales en las capas epidérmicas superiores y tendencia a la vacuolización basal ${ }^{11}$, como el caso informado. Esta lamela como hallazgo en la histopatología de un patrón acantolítico focal se considera una variante histopatológica poco frecuente de esta dermatosis, denominada patrón poroqueratósico.

Ante una clínica y dermatoscopia indicativa de enfermedad de Grover se debe realizar una correlación con el estudio histopatológico, que muestre acantólisis focal y en el caso de la variante poroqueratósica columnas de paraqueratosis ${ }^{12}$.

Se presentó el caso de una mujer de 27 años de edad con manifestaciones clínicas y dermatoscópicas típicas de la enfermedad de Grover junto con una histopatología que confirmó el diagnóstico y reveló una variante infrecuente, de tipo poroqueratósico, que debe considerarse al observar una lamela cornoide junto al hallazgo característico de la enfermedad de Grover, la acantólisis focal discreta.

\section{Conclusiones}

La correlación clínica-patológica en esta entidad es importante para un diagnóstico y tratamiento adecuado. El conocimiento de los patrones habituales y atípicos contribuye a una interpretación adecuada del diagnóstico y a comprender la patogenia de esta enfermedad acantolítica. Una acantólisis focal discreta vinculada con una lamela cornoide debe orientar al diagnóstico de enfermedad de Grover de tipo poroqueratósico y su escasa frecuencia hace relevante este caso para las publicaciones médicas y conocimiento médico.

\section{Financiamiento}

Los autores declaran no haber recibido financiamiento para este estudio.

\section{Conflicto de intereses}

Los autores declaran no tener conflicto de intereses.

\section{Responsabilidades éticas}

Protección de personas y animales. Los autores declaran que para esta investigación no se han realizado experimentos en seres humanos ni en animales.

Confidencialidad de los datos. Los autores declaran que han seguido los protocolos de su centro de trabajo sobre la publicación de datos de pacientes.

Derecho a la privacidad y consentimiento informado. Los autores han obtenido el consentimiento informado de los pacientes y/o sujetos referidos en el 
artículo. Este documento obra en poder del autor de correspondencia.

\section{Bibliografía}

1. Grover RW, Park F. Transient acantholytic dermatosis. Arch Derm. 1970;101.

2. Grover R, Ackerman AB. Transient acantholytic dermatosis a reevaluation. Arch Dermatol. 1977:113:431-135.

3. Scheinfeld N, Mones J. Seasonal variation of transient acantholytic dyskeratosis (Grover's disease). J Am Acad Dermatol. 2006;55(2):263-8.

4. Davis MDP, Dinneen AM, Landa N, Gibson LE. Grover's disease: clinicopathologic review of 72 cases. Mayo Clin Proc. 1999;74(3):229-34.

5. Pasmatzi E, Kousparos G, Mytidou C, Nicolaou N, Tanteles GA, Badavanis $\mathrm{G}$, et al. Persistent generalized Grover disease: complete remission after treatment with oral acitretin. Dermatol Online J. 2019;25.
6. Quirk CJ, Heenan PJ. Grover's disease: 34 years on. Australas J Dermatol. 2004;45(2):83-8.

7. Gantz M, Butler D, Goldberg M, Ryu J, McCalmont T, Shinkai K. Atypical features and systemic associations in extensive cases of Grover disease: a systematic review. J Am Acad Dermatol [Internet]. 2017;77(5):952-957. e1. Available from: $h$ ttp://dx.doi.org/10.1016/i.jaad.2017.06.041

8. de Abreu L, Guimarães-Cordeiro NG, Buçard AM, Quintella DC, Argenziano G. Dermoscopy of Grover disease. J Am Acad Dermatol. 2017;76(2):S60-3.

9. Errichetti $E$, Stinco G. Dermoscopy in general dermatology: a practical overview. vol. 6, dermatology and therapy. Springer Healthcare. 2016;471-507.

10. Fernández-Figueras MT, Puig L, Cannata $P$, Cuatrecases $M$, Quer $A$, Ferrándiz $C$, et al. Grover disease: a reappraisal of histopathological diagnostic criteria in 120 cases. Am J Dermatopathol. 2010;32(6):541-9.

11. Aljarbou OZ, Asgari M, Al-Saidi N, Silloca-Cabana EO, Alathamneh M, Sangueza OP. Grover disease with epidermal dysmaturation pattern: a common histopathologic finding. Am J Dermatopathol. 2018;40(9):642-6.

12. Montoya C, Arias LM, Salazar M, Flórez HA. Porokeratosis-like Grover disease: more than an acantholytic pattern. Actas Dermosifiliogr. 2019;110(4):332-4. 